\title{
On Stochastic Differential Equations with Reflecting Boundary Condition in Convex Domains
}

by

\author{
Weronika ŁAUKAJTYS
}

Presented by Jerzy $Z A B C Z Y K$

Summary. Let $D$ be an open convex set in $\mathbb{R}^{d}$ and let $F$ be a Lipschitz operator defined on the space of adapted càdlàg processes. We show that for any adapted process $H$ and any semimartingale $Z$ there exists a unique strong solution of the following stochastic differential equation (SDE) with reflection on the boundary of $D$ :

$$
X_{t}=H_{t}+\int_{0}^{t}\left\langle F(X)_{s-}, d Z_{s}\right\rangle+K_{t}, \quad t \in \mathbb{R}^{+} .
$$

Our proofs are based on new a priori estimates for solutions of the deterministic Skorokhod problem.

1. Introduction. In the present paper we consider the following SDE with reflection on the boundary $\partial D$ of an open convex set $D \subset \mathbb{R}^{d}$ :

$$
X_{t}=H_{t}+\int_{0}^{t}\left\langle F(X)_{s-}, d Z_{s}\right\rangle+K_{t}, \quad t \in \mathbb{R}^{+} .
$$

Here $Z=\left(Z_{t}\right)$ is an $\left(\mathcal{F}_{t}\right)$ adapted semimartingale with $Z_{0}=0, H=\left(H_{t}\right)$ is an $\left(\mathcal{F}_{t}\right)$ adapted process with $H_{0} \in \bar{D}=D \cup \partial D$ and $F$ is a Lipschitz operator on the space of adapted càdlàg processes (for a precise definition see Section 3).

The problem of existence and uniqueness of solutions of (1.1) was discussed for the first time by Skorokhod [7] in the case where $d=1, D=$

2000 Mathematics Subject Classification: Primary 60H20.

Key words and phrases: stochastic differential equation with reflecting boundary, Skorokhod problem.

Research supported by Komitet Badań Naukowych under grant 1 P03A 02226. 
$(0, \infty), H=X_{0} \in[0, \infty)$ and $Z$ is a standard Wiener process $W$. Next, many attempts have been made to generalize Skorokhod's results to a larger class of domains or a larger class of driving processes $Z$. In particular, existence and uniquness of solutions of (1.1) with $Z=W$ for an arbitrary convex set $D$ was proved by Tanaka [11]. SDEs driven by general semimartingales were considered in detail by Słomiński $[8,9]$. Let us stress, however, that in the above mentioned papers $[8,9,11]$ it is assumed that $H=X_{0}$ and that $F(X)_{s-}=f\left(X_{s-}\right)$, where $f$ is a Lipschitz continuous function.

In the present paper we show existence and uniqueness of solutions of the SDE (1.1) for an arbitrary $\left(\mathcal{F}_{t}\right)$ adapted process $H=\left(H_{t}\right)$ with $H_{0} \in \bar{D}$ and an arbitrary Lipschitz operator $F$, and thus we generalize the results of $[8,9,11]$ considerably. The proof of our main result is based on new a priori estimates for the solution of the deterministic Skorokhod problem

$$
x_{t}=y_{t}+k_{t}, \quad t \in \mathbb{R}^{+},
$$

associated with a $y \in \mathbb{D}\left(\mathbb{R}^{+}, \mathbb{R}^{d}\right)$ such that $y_{0} \in \bar{D}$. These estimates say that for any $T \in \mathbb{R}^{+}$and any $a \in D, \sup _{t \leq T}\left|x_{t}-a\right|$ and $|k|_{T}$ are bounded by constants depending only on $y_{0}, \sup _{t \leq T}\left|y_{t}-a\right|$ and the modulus of continuity $\omega_{y}^{\prime}$. As a consequence, we prove existence and uniqueness of solutions of the Skorokhod problem (1.2) in an arbitrary open convex set $D$. In this way we solve Tanaka's problem (see [11, Remark 2.3]) concerning existence of a solution of the Skorokhod problem associated with $y \in \mathbb{D}\left(\mathbb{R}^{+}, \mathbb{R}^{d}\right.$ ) (in the case where $y$ is continuous, Tanaka's problem was solved earlier by Cépa [2]).

Notation. $\mathbb{D}\left(\mathbb{R}^{+}, \mathbb{R}^{d}\right)$ is the space of all mappings $y: \mathbb{R}^{+} \rightarrow \mathbb{R}^{d}$ which are right-continuous and admit left-hand limits. Every process appearing in what follows is assumed to have its trajectories in the space $\mathbb{D}\left(\mathbb{R}^{+}, \mathbb{R}^{d}\right)$ endowed with the Skorokhod topology $J_{1}$. If $X=\left(X^{1}, \ldots, X^{d}\right)$ is a semimartingale then $[X]_{t}=\sum_{i=1}^{d}\left[X^{i}\right]_{t}$, where $\left[X^{i}\right]$ stands for the quadratic variation process of $X^{i}, i=1, \ldots, d$. Similarly, $\langle X\rangle_{t}=\sum_{i=1}^{d}\left\langle X^{i}\right\rangle_{t}$, where $\left\langle X^{i}\right\rangle_{t}$ stands for the predictable compensator of $\left[X^{i}\right], i=1, \ldots, d$. If $K=\left(K^{1}, \ldots, K^{d}\right)$ is a process with locally finite variation, then $|K|_{t}=$ $\sum_{i=1}^{d}\left|K^{i}\right|_{t}$, where $\left|K^{i}\right|_{t}$ is the total variation of $K^{i}$ on $[0, t]$. For $x \in$ $\mathbb{D}\left(\mathbb{R}^{+}, \mathbb{R}^{d}\right), \delta>0, T \in \mathbb{R}^{+}$we denote by $\omega_{x}^{\prime}(\delta, T)$ the modulus of continuity of $x$ on $[0, T]$, i.e.

$\omega_{x}^{\prime}(\delta, T)=\inf \left\{\max _{i \leq r} \omega_{x}\left(\left[t_{i-1}, t_{i}\right)\right) ; 0=t_{0}<\cdots<t_{r}=T, \inf _{i<r}\left(t_{i}-t_{i-1}\right) \geq \delta\right\}$, where $\omega_{x}(I)=\sup _{s, t \in I}\left|x_{s}-x_{t}\right|$.

2. The Skorokhod problem. Let $D$ be an open convex domain in $\mathbb{R}^{d}$ and let $\mathcal{N}_{x}$ denote the set of inward normal unit vectors at $x \in \partial D$.

The following remark can be found in Menaldi [4] and Storm [10]. 
REMARK 2.1. (i) $\mathbf{n} \in \mathcal{N}_{x}$ if and only if $\langle y-x, \mathbf{n}\rangle \geq 0$ for every $y \in \bar{D}$, where $\langle\cdot, \cdot\rangle$ denotes the usual inner product in $\mathbb{R}^{d}$.

(ii) If $\operatorname{dist}(x, \bar{D})>0$, then there exists a unique $\Pi(x) \in \partial D$ such that $|\Pi(x)-x|=\operatorname{dist}(x, \bar{D})$. Moreover, $(\Pi(x)-x) /|\Pi(x)-x| \in \mathcal{N}_{\Pi(x)}$.

(iii) For every $a \in D$ and $\mathbf{n} \in \mathcal{N}_{x}$,

$$
\langle x-a, \mathbf{n}\rangle \leq-\operatorname{dist}(a, \partial D) .
$$

Let $y \in \mathbb{D}\left(\mathbb{R}^{+}, \mathbb{R}^{d}\right)$ with $y_{0} \in \bar{D}$. We say that a pair $(x, k)$ is a solution of the Skorokhod problem associated with $y$ if

$$
\begin{aligned}
& \text { (2.1) } x_{t}=y_{t}+k_{t}, t \in \mathbb{R}^{+}, \\
& (2.2) \quad x_{t} \in \bar{D}, t \in \mathbb{R}^{+}, \\
& \text {(2.3) } k \text { is a function with locally bounded variation, } k_{0}=0 \text {, and }
\end{aligned}
$$

$$
k_{t}=\int_{0}^{t} \mathbf{n}_{s} d|k|_{s}, \quad|k|_{t}=\int_{0}^{t} \mathbf{1}_{\left\{x_{s} \in \partial D\right\}} d|k|_{s}, \quad t \in \mathbb{R}^{+},
$$

where $\mathbf{n}_{s} \in \mathcal{N}_{x_{s}}$ if $x_{s} \in \partial D$.

The following estimates on the solution of the Skorokhod problem will prove extremely useful in the proofs of our main results.

THEOREM 2.2. Let $(x, k)$ be a solution of the Skorokhod problem associated with $y$, and let $y_{0} \in \bar{D}$. Then for any $T>0, \eta>0$ and $a \in D$ such that

$$
\omega_{y}^{\prime}(\eta, T)<\frac{\operatorname{dist}(a, \partial D)}{2}
$$

we have

(i) $\sup _{t \leq T}\left|x_{t}-a\right| \leq 6([T / \eta]+1) \sup _{t \leq T}\left|y_{t}-a\right|$,

(ii) $|k|_{T} \leq \frac{71([T / \eta]+1)^{3}}{\operatorname{dist}(a, \partial D)} \sup _{t \leq T}\left|y_{t}-a\right|^{2}$

$([T / \eta]$ denotes the largest integer less than or equal to $T / \eta)$.

Proof. (i) We proceed along the lines of the proof of Theorem 3.2 in [2]. Let $0 \leq t \leq T$. It is easily seen that

$$
\begin{aligned}
\left|x_{t}-a\right|^{2} & =\left|y_{t}-a\right|^{2}+\left\langle k_{t}, k_{t}\right\rangle+2 \int_{0}^{t}\left\langle y_{t}-a, d k_{u}\right\rangle \\
& =\left|y_{t}-a\right|^{2}+2 \int_{0}^{t}\left\langle k_{u}, d k_{u}\right\rangle-\sum_{u \leq t}\left|\Delta k_{u}\right|^{2}+2 \int_{0}^{t}\left\langle y_{t}-a, d k_{u}\right\rangle \\
& =\left|y_{t}-a\right|^{2}+2 \int_{0}^{t}\left\langle x_{u}-a, d k_{u}\right\rangle+2 \int_{0}^{t}\left\langle y_{t}-y_{u}, d k_{u}\right\rangle-\sum_{u \leq t}\left|\Delta k_{u}\right|^{2} .
\end{aligned}
$$


Therefore, for any $0 \leq s \leq t \leq T$,

$$
\begin{aligned}
\left|x_{t}-a\right|^{2}-\left|x_{s}-a\right|^{2}= & \left|y_{t}-a\right|^{2}-\left|y_{s}-a\right|^{2}+2 \int_{s}^{t}\left\langle x_{u}-a, d k_{u}\right\rangle \\
& -2 \int_{s}^{t}\left\langle y_{u}-y_{s}, d k_{u}\right\rangle+2\left\langle k_{t}, y_{t}-y_{s}\right\rangle-\sum_{s<u \leq t}\left|\Delta k_{u}\right|^{2} .
\end{aligned}
$$

By Remark 2.1(iii), $2 \int_{s}^{t}\left\langle x_{u}-a, d k_{u}\right\rangle \leq-2 \operatorname{dist}(a, \partial D)|k|_{s}^{t}$, where $|k|_{s}^{t}=$ $|k|_{t}-|k|_{s}$. Hence

$$
\begin{aligned}
\left|x_{t}-a\right|^{2}-\left|x_{s}-a\right|^{2} \leq & \left|y_{t}-a\right|^{2}-\left|y_{s}-a\right|^{2}-2 \operatorname{dist}(a, \partial D)|k|_{s}^{t} \\
& \quad-2 \int_{s}^{t}\left\langle y_{u}-y_{s}, d k_{u}\right\rangle-2\left\langle y_{t}-a, y_{t}-y_{s}\right\rangle \\
& -2\left\langle a-x_{t}, y_{t}-y_{s}\right\rangle-\sum_{s<u \leq t}\left|\Delta k_{u}\right|^{2} \\
\leq & 5 \sup _{t \leq T}\left|y_{t}-a\right|^{2}+4 \sup _{t \leq T}\left|y_{t}-a\right| \cdot \sup _{t \leq T}\left|x_{t}-a\right| \\
& -2 \operatorname{dist}(a, \partial D)|k|_{s}^{t}-2 \int_{s}^{t}\left\langle y_{u}-y_{s}, d k_{u}\right\rangle-\sum_{s<u \leq t}\left|\Delta k_{u}\right|^{2} .
\end{aligned}
$$

On the other hand, since $y \in \mathbb{D}\left(\mathbb{R}^{+}, \mathbb{R}^{d}\right)$ it follows that there exist $\eta>0$ and a subdivision $\left(s_{k}\right)$ of $[0, T]$ such that $0=s_{0}<s_{1}<\cdots<s_{r}=T$, $\eta \leq s_{k}-s_{k-1}, k=1, \ldots, r-1$, where $r=[T / \eta]+1$, and

$$
\omega_{y}\left(\left[s_{k-1}, s_{k}\right)\right)<\frac{\operatorname{dist}(a, \partial D)}{2} .
$$

Using (2.5) we obtain

$$
\begin{aligned}
-\int_{s_{k-1}}^{s_{k}}\left\langle y_{u}-y_{s_{k-1}}, d k_{u}\right\rangle & \leq\left|\int_{\left(s_{k-1}, s_{k}\right)}\left\langle y_{u}-y_{s_{k-1}}, d k_{u}\right\rangle\right|-\left\langle\Delta y_{s_{k}}, \Delta k_{s_{k}}\right\rangle \\
& \leq \frac{\operatorname{dist}(a, \partial D)}{2}|k|_{s_{k-1}}^{s_{k}}-\left\langle\Delta y_{s_{k}}, \Delta k_{s_{k}}\right\rangle .
\end{aligned}
$$

Therefore

$$
\begin{aligned}
2\left(-\int_{s_{k-1}}^{s_{k}}\langle\right. & \left.\left.y_{u}-y_{s_{k-1}}, d k_{u}\right\rangle-\operatorname{dist}(a, \partial D)|k|_{s_{k-1}}^{s_{k}}\right) \\
& \leq 2\left(\frac{\operatorname{dist}(a, \partial D)}{2}|k|_{s_{k-1}}^{s_{k}}-\operatorname{dist}(a, \partial D)|k|_{s_{k-1}}^{s_{k}}-\left\langle\Delta y_{s_{k}}, \Delta k_{s_{k}}\right\rangle\right) \\
& =-\operatorname{dist}(a, \partial D)|k|_{s_{k-1}}^{s_{k}}-2\left\langle\Delta y_{s_{k}}, \Delta k_{s_{k}}\right\rangle
\end{aligned}
$$


and, as a consequence,

$$
\begin{aligned}
& \leq 5 \sup _{t \leq T}\left|y_{t}-a\right|^{2}+4 \sup _{t \leq T}\left|y_{t}-a\right| \cdot \sup _{t \leq T}\left|x_{t}-a\right| \\
& \quad-\operatorname{dist}(a, \partial D)|k|_{s_{k-1}}^{s_{k}}-2\left\langle\Delta y_{s_{k}}, \Delta k_{s_{k}}\right\rangle-\sum_{s_{k-1}<u \leq s_{k}}\left|\Delta k_{u}\right|^{2} \\
& \leq 5 \sup _{t \leq T}\left|y_{t}-a\right|^{2}+4 \sup _{t \leq T}\left|y_{t}-a\right| \cdot \sup _{t \leq T}\left|x_{t}-a\right|-\operatorname{dist}(a, \partial D)|k|_{s_{k-1}}^{s_{k}}+\left|\Delta y_{s_{k}}\right|^{2} \\
& \leq 9 \sup _{t \leq T}\left|y_{t}-a\right|^{2}+4 \sup _{t \leq T}\left|y_{t}-a\right| \cdot \sup _{t \leq T}\left|x_{t}-a\right|-\operatorname{dist}(a, \partial D)|k|_{s_{k-1}}^{s_{k}} .
\end{aligned}
$$

From (2.6) it follows immediately that

$$
\left|x_{s_{k}}-a\right|^{2}-\left|x_{s_{k-1}}-a\right|^{2} \leq 9 \sup _{t \leq T}\left|y_{t}-a\right|^{2}+4 \sup _{t \leq T}\left|y_{t}-a\right| \cdot \sup _{t \leq T}\left|x_{t}-a\right| .
$$

For given $t \in[0, T]$ set $k_{0}=\max \left\{k ; s_{k} \leq t\right\}$. Then

$$
\begin{aligned}
\left|x_{t}-a\right|^{2} & =\sum_{k=1}^{k_{0}}\left(\left|x_{s_{k}}-a\right|^{2}-\left|x_{s_{k-1}}-a\right|^{2}\right)+\left|x_{t}-a\right|^{2}-\left|x_{s_{k_{0}}}-a\right|^{2}+\left|x_{0}-a\right|^{2} \\
& \leq r\left(9 \sup _{t \leq T}\left|y_{t}-a\right|^{2}+4 \sup _{t \leq T}\left|y_{t}-a\right| \cdot \sup _{t \leq T}\left|x_{t}-a\right|\right)+\sup _{t \leq T}\left|y_{t}-a\right|^{2},
\end{aligned}
$$

which implies that

$$
\sup _{t \leq T}\left|x_{t}-a\right|^{2} \leq 18 r^{2} \sup _{t \leq T}\left|y_{t}-a\right|^{2}+\sup _{t \leq T}\left|x_{t}-a\right|^{2} / 2 .
$$

Hence

$$
\sup _{t \leq T}\left|x_{t}-a\right|^{2} \leq 36 r^{2} \sup _{t \leq T}\left|y_{t}-a\right|^{2}
$$

and the proof of (i) is complete.

(ii) Using (2.6) and (2.7) gives

$$
\begin{aligned}
\operatorname{dist}(a, \partial D)|k|_{s_{k-1}}^{s_{k}} \leq & 9 \sup _{t \leq T}\left|y_{t}-a\right|^{2}+4 \sup _{t \leq T}\left|y_{t}-a\right| \cdot \sup _{t \leq T}\left|x_{t}-a\right| \\
& +\left|x_{s_{k-1}}-a\right|^{2}-\left|x_{s_{k}}-a\right|^{2} \\
\leq & 17 \sup _{t \leq T}\left|y_{t}-a\right|^{2}+\frac{3}{2} \sup _{t \leq T}\left|x_{t}-a\right|^{2} \leq 71 r^{2} \sup _{t \leq T}\left|y_{t}-a\right|^{2}
\end{aligned}
$$

for $k=1, \ldots, r$. Since $|k|_{T} \leq \sum_{k=1}^{r}|k|_{s_{k-1}}^{s_{k}}$, this proves (ii).

Corollary 2.3. If $\left\{y^{n}\right\}$ is relatively compact in $\mathbb{D}\left(\mathbb{R}^{+}, \mathbb{R}^{d}\right)$ then

(i) $\sup _{n} \sup _{t \leq T}\left|x_{t}^{n}\right|<\infty$ and $\sup _{n}\left|k^{n}\right|_{T}<\infty$ for every $T>0$,

(ii) $\left\{\left(x^{n}, k^{n}\right)\right\}$ is relatively compact in $\mathbb{D}\left(\mathbb{R}^{+}, \mathbb{R}^{2 d}\right)$. 
Proof. (i) Clearly, $\sup _{n}\left|x_{0}^{n}\right|=\sup _{n}\left|y_{0}^{n}\right|<\infty$. Since $\left\{y^{n}\right\}$ is relatively compact, for any $a \in D, T>0$ there exists $\eta>0$ such that $\sup _{n} \omega_{y^{n}}^{\prime}(\eta, T)$ $<\operatorname{dist}(a, \partial D) / 2$. Moreover, relative compactness of $\left\{y^{n}\right\}$ implies that $\sup _{n} \sup _{t \leq T}\left|y_{t}^{n}\right|<\infty$. Therefore (i) follows from Theorem 2.2.

(ii) Since $\left\{y^{n}\right\}$ is relatively compact, for any $T>0$ and $\varepsilon>0$ there exist $\delta>0$ and $0=s_{0}<s_{1}<\cdots<s_{r}=T$ such that $\delta \leq s_{k}-s_{k-1}$ and $\omega_{y^{n}}\left(\left[s_{k-1}, s_{k}\right)\right) \leq \varepsilon$ for $k=1, \ldots, r-1$. By [11, Lemma 2.2], for $n \in \mathbb{N}$ and $t, s \in \mathbb{R}^{+}$we have

$$
\left|x_{t}^{n}-x_{s}^{n}\right|^{2} \leq\left|y_{t}^{n}-y_{s}^{n}\right|^{2}+\int_{s}^{t}\left\langle y_{t}^{n}-y_{u}^{n}, d k_{u}^{n}\right\rangle .
$$

Therefore

$$
\sup _{s_{k-1} \leq t<s_{k}}\left|x_{t}^{n}-x_{s_{k-1}}^{n}\right|^{2} \leq \omega_{y^{n}}^{2}\left(\left[s_{k-1}, s_{k}\right)\right)+\omega_{y^{n}}\left(\left[s_{k-1}, s_{k}\right)\right)\left|k^{n}\right|_{T} \leq \varepsilon\left(\varepsilon+\left|k^{n}\right|_{T}\right),
$$

and hence, $\max _{k \leq r} \omega_{x^{n}}\left(\left[s_{k-1}, s_{k}\right)\right) \leq\left(\varepsilon\left(\varepsilon+\left|k^{n}\right|_{T}\right)\right)^{1 / 2}$. As a consequence,

$$
\lim _{\delta \downarrow 0} \sup _{n} \omega_{\left(x^{n}, y^{n}\right)}^{\prime}(\delta, T)=0,
$$

which together with (i) shows that $\left\{\left(x^{n}, y^{n}\right)\right\}$ is relatively compact in $\mathbb{D}\left(\mathbb{R}^{+}, \mathbb{R}^{2 d}\right)$. Since $k^{n}=x^{n}-y^{n}$, (ii) follows.

Corollary 2.4. Let $(x, k)$ be a solution of the Skorokhod problem associated with y such that $y_{0} \in \bar{D}$, and let $a \in D$. Set $\tau=\inf \left\{t>0 ;\left|y_{t}-y_{0}\right| \geq\right.$ $\operatorname{dist}(a, \partial D) / 2\}$. Then

$$
\sup _{t<\tau}\left|x_{t}-a\right| \leq 6 \sup _{t<\tau}\left|y_{t}-a\right|, \quad|k|_{\tau-} \leq \frac{71}{\operatorname{dist}(a, \partial D)} \sup _{t<\tau}\left|y_{t}-a\right|^{2} .
$$

Proof. It suffices to put $r=1, s_{0}=0, s_{1}=\tau$ in the proof of Theorem 2.2 .

Theorem 2.5. Assume $\left\{y^{n}\right\} \subset \mathbb{D}\left(\mathbb{R}^{+}, \mathbb{R}^{d}\right), y_{0}^{n} \in \bar{D}$ and let $\left(x^{n}, k^{n}\right)$ denote the solution of the Skorokhod problem associated with $y^{n}, n \in \mathbb{N}$. If $y^{n} \rightarrow y$ in $\mathbb{D}\left(\mathbb{R}^{+}, \mathbb{R}^{d}\right)$ and $y_{0} \in \bar{D}$ then there exists a unique solution $(x, k)$ of the Skorokhod problem associated with $y$ and

$$
\left(x^{n}, k^{n}\right) \rightarrow(x, k) \quad \text { in } \mathbb{D}\left(\mathbb{R}^{+}, \mathbb{R}^{2 d}\right) .
$$

Proof. By Corollary 2.3(ii), the sequence $\left\{\left(x^{n}, y^{n}, k^{n}\right)\right\}$ is relatively compact in $\mathbb{D}\left(\mathbb{R}^{+}, \mathbb{R}^{3 d}\right)$. Therefore, there exists a subsequence $\left(n^{\prime}\right) \subset(n)$ and a pair $\left(x^{\prime}, k^{\prime}\right)$ such that

$$
\left(x^{n^{\prime}}, y^{n^{\prime}}, k^{n^{\prime}}\right) \rightarrow\left(x^{\prime}, y, k^{\prime}\right) \quad \text { in } \mathbb{D}\left(\mathbb{R}^{+}, \mathbb{R}^{3 d}\right) .
$$

By [11, Lemma 2.2] the solution of the Skorokhod problem associated with $y$ is unique. Therefore, the proof is completed by showing that $\left(x^{\prime}, k^{\prime}\right)$ is a solution of the Skorokhod problem. Obviously $x^{\prime}=y+k^{\prime}$. Moreover, by Corollary 2.3(i), $\sup _{n}\left|k^{n}\right|_{T}<\infty, T \in \mathbb{R}^{+}$, which implies that $\left|k^{\prime}\right|_{T}<\infty$, 
$T \in \mathbb{R}^{+}$. To check (2.3), we first note that it is equivalent to the following two conditions: for any bounded continuous $f: \bar{D} \rightarrow \mathbb{R}^{d}$ such that $f(x)=0$ for $x \in \partial D$ we have

$$
\int_{0}^{t}\left\langle f\left(x_{s}\right), d k_{s}\right\rangle=0, \quad t \in \mathbb{R}^{+},
$$

and for any continuous $\widehat{x}: \mathbb{R}^{+} \rightarrow \bar{D}$ the function

$$
t \mapsto \int_{0}^{t}\left\langle\widehat{x}_{s}-x_{s}, d k_{s}\right\rangle, t \in \mathbb{R}^{+}, \text {is non-decreasing }
$$

(see e.g. [1]).

By (2.8) and [3, Proposition 2.9],

$$
\int_{0}^{t}\left\langle f\left(x_{s}^{n^{\prime}}\right), d k_{s}^{n^{\prime}}\right\rangle \rightarrow \int_{0}^{t}\left\langle f\left(x_{s}^{\prime}\right), d k_{s}^{\prime}\right\rangle \quad \text { in } \mathbb{D}\left(\mathbb{R}^{+}, \mathbb{R}\right) .
$$

On the other hand, since $\left(x^{n^{\prime}}, k^{n^{\prime}}\right)$ is a solution of the Skorokhod problem, for each $n^{\prime}$ we have $\int_{0}^{t}\left\langle f\left(x_{s}^{n^{\prime}}\right), d k_{s}^{n^{\prime}}\right\rangle=0$. Therefore (2.11) gives (2.9). Furthermore, by $(2.8),\left(\widehat{x}, x^{n^{\prime}}, k^{n^{\prime}}\right) \rightarrow\left(\widehat{x}, x^{\prime}, k^{\prime}\right)$ in $\mathbb{D}\left(\mathbb{R}^{+}, \mathbb{R}^{3 d}\right)$. Hence, using once again [3, Proposition 2.9], we obtain

$$
\int_{0}^{t}\left\langle\widehat{x}_{s}-x_{s}^{n^{\prime}}, d k_{s}^{n^{\prime}}\right\rangle \rightarrow \int_{0}^{t}\left\langle\widehat{x}_{s}-x_{s}^{\prime}, d k_{s}^{\prime}\right\rangle \quad \text { in } \mathbb{D}\left(\mathbb{R}^{+}, \mathbb{R}\right),
$$

which implies (2.10), because the functions $t \mapsto \int_{0}^{t}\left\langle\widehat{x}_{s}-x_{s}^{n^{\prime}}, d k_{s}^{n^{\prime}}\right\rangle$ are nondecreasing.

Theorem 2.6. For every $y \in \mathbb{D}\left(\mathbb{R}^{+}, \mathbb{R}^{d}\right)$ such that $y_{0} \in \bar{D}$ there exists a unique solution of the Skorokhod problem associated with $y$.

Proof. Let $\left\{y^{n}\right\}$ be the sequence of discretizations of $y$ defined by $y_{t}^{n}=$ $y_{k / n}, t \in[k / n,(k+1) / n), n \in \mathbb{N}$. We check at once that for every $n \in \mathbb{N}$ the pair $\left(x^{n}, k^{n}\right)$ defined by

$$
\left\{\begin{array}{l}
x_{0}^{n}=y_{0}, \\
x_{(k+1) / n}^{n}=\Pi\left(x_{k / n}^{n}+y_{(k+1) / n}-y_{k / n}\right)
\end{array}\right.
$$

and $x_{t}^{n}=x_{k / n}^{n}, k_{t}^{n}=x_{t}^{n}-y_{t}^{n}, t \in[k / n,(k+1) / n), n \in \mathbb{N}$, solves the Skorokhod problem for $y^{n}$. Since $y^{n} \rightarrow y$ in $\mathbb{D}\left(\mathbb{R}^{+}, \mathbb{R}^{d}\right)$, the result follows from Theorem 2.5.

3. SDEs in convex domains. Let $\left(\Omega, \mathcal{F},\left(\mathcal{F}_{t}\right), P\right)$ be a filtered probability space and let $Y$ be an $\left(\mathcal{F}_{t}\right)$ adapted process with $Y_{0} \in \bar{D}$. 
We say that a pair $(X, K)$ of $\left(\mathcal{F}_{t}\right)$ adapted processes solves the Skorokhod problem associated with $Y$ if for almost every $\omega \in \Omega$ the pair $(X(\omega), K(\omega))$ is a solution of the Skorokhod problem associated with $Y(\omega)$.

From Theorem 2.6 it follows that for any process $Y$ with $Y_{0} \in \bar{D}$ there exists a unique solution of the Skorokhod problem associated with $Y$. The following remark is due to Słomiński (see [8, Corollary 1]).

Remark 3.1. Let $Y, \widehat{Y}$ be $\left(\mathcal{F}_{t}\right)$ adapted processes of the form $Y=$ $H+M+V, \widehat{Y}=H+\widehat{M}+\widehat{V}$, where $M, \widehat{M}$ are local martingales, $V, \widehat{V}$ are processes with locally bounded variation and $M_{0}=\widehat{M}_{0}=V_{0}=\widehat{V}_{0}=0$. If $(X, K),(\widehat{X}, \widehat{K})$ are solutions of the Skorokhod problem associated with $Y, \widehat{Y}$, respectively, then for every $p \in \mathbb{N}$ there exists $C_{p}$ such that

$$
E \sup _{t<\tau}\left|X_{t}-\widehat{X}_{t}\right|^{2 p} \leq C_{p} E\left([M-\widehat{M}]_{\tau-}^{p}+|V-\widehat{V}|_{\tau-}^{2 p}+\langle M-\widehat{M}\rangle_{\tau-}^{p}\right)
$$

for every $\left(\mathcal{F}_{t}\right)$ stopping time $\tau$.

Let us denote by $\mathbb{F}^{d}$ the class of $d$-dimensional $\left(\mathcal{F}_{t}\right)$ adapted processes and by $\mathbb{M}^{d}$ the class of $\left(\mathcal{F}_{t}\right)$ adapted processes with values in the set $\mathbb{R}^{d} \otimes \mathbb{R}^{d}$ of $d$-dimensional matrices.

We say that an operator $F: \mathbb{F}^{d} \rightarrow \mathbb{M}^{d}$ is Lipschitz if

(i) for every $X, Y \in \mathbb{F}^{d}$ and every stopping time $\tau$, if $X^{\tau-}=Y^{\tau-}$ then $F(X)^{\tau-}=F(Y)^{\tau-}$

(ii) there exists a one-dimensional, $\left(\mathcal{F}_{t}\right)$ adapted, non-decreasing process $L=\left(L_{t}\right)$ such that $P\left(\sup _{t} L_{t}<\infty\right)=1$ and, for every $X, Y \in \mathbb{F}^{d}$,

$$
\left\|F(X)_{t}-F(Y)_{t}\right\| \leq L_{t} \sup _{s \leq t}\left|X_{s}-Y_{s}\right|, \quad t \in \mathbb{R}^{+} .
$$

Clearly, if $f: \mathbb{R}^{d} \rightarrow \mathbb{R}^{d} \otimes \mathbb{R}^{d}$ is Lipschitz, that is, there exists $L>0$ such that $\|f(x)-f(y)\| \leq L|x-y|$ for $x, y \in \mathbb{R}^{d}$, then the operator $F(X)$ defined by $F(X)_{t}=f\left(X_{t}\right)$ for $X \in \mathbb{F}^{d}$ is Lipschitz.

Let $F: \mathbb{F}^{d} \rightarrow \mathbb{M}^{d}$. We say that a pair $(X, K)$ of $\left(\mathcal{F}_{t}\right)$ adapted processes is a strong solution of the $\mathrm{SDE}$ (1.1) if (1.1) is satisfied and $(X, K)$ is a solution of the Skorokhod problem associated with $Y_{t}=H_{t}+\int_{0}^{t}\left\langle F(X)_{s-}, d Z_{s}\right\rangle$, $t \in \mathbb{R}^{+}$.

We can now formulate our main result.

Theorem 3.2. Let $H$ be an $\left(\mathcal{F}_{t}\right)$ adapted process such that $H_{0} \in \bar{D}$, and let $Z$ be an $\left(\mathcal{F}_{t}\right)$ adapted semimartingale with $Z_{0}=0$. Then for any Lipschitz operator $F: \mathbb{F}^{d} \rightarrow \mathbb{M}^{d}$ there exists a unique strong solution of the SDE (1.1).

Proof. By using the arguments from the proof of $[6$, Chapter V, Theorem 7], we may and do assume that $F(0)_{t}=0$ and $L_{t}<L$ for some constant 
$L>0$. Since $Z$ is a semimartingale, it admits a unique decomposition

$$
Z_{t}=J_{t}+M_{t}+B_{t}, \quad t \in \mathbb{R}^{+},
$$

where $\left.J_{t}=\sum_{s \leq t} \Delta Z_{u} \mathbb{I}\left\{\left|\Delta Z_{s}\right|>1\right]\right\}, M$ is a local square-integrable martingale, $|\Delta M| \leq 2$, and $B$ is a predictable process with locally bounded variation, $|\Delta B| \leq 1$. Let $C_{1}$ be a constant from Remark 3.1. For given $a^{\prime} \in D$ set $a^{2}=\left(12 C_{1} L^{2}\right)^{-1}$ and $\tau^{\prime}=\inf \left\{t>0 ;\left|H_{t}-H_{0}\right| \geq \operatorname{dist}\left(a^{\prime}, \partial D\right) / 2\right\}$.

We first prove existence and uniqueness of a solution of (1.1) on the interval $\left[0, \tau\left[\right.\right.$, where $\tau=\inf \left\{t>0 ; \max \left([M]_{t},\langle M\rangle_{t},|B|_{t}^{2},|J|_{t}^{2}\right)>a^{2}\right\} \wedge \tau^{\prime} \wedge 1$. To this end we set $\tau_{k}=\inf \left\{t>0 ; \sup _{s \leq t}\left|H_{s}\right|>k\right\} \wedge \tau$ for $k \in \mathbb{N}$. For fixed $k \in \mathbb{N}$ we denote by $S^{2}$ the class of $\left(\mathcal{F}_{t}\right)$ adapted processes $Y=\left(Y_{t}\right)$ on $[0,1]$ such that $Y_{0} \in \bar{D}, Y=Y^{\tau_{k}-}$ and $E \sup _{t \leq 1}\left|Y_{t}\right|^{2}<\infty$. Then $S^{2}$ is a Banach space with the norm $\|Y\|_{S^{2}}=\left(E \sup _{t \leq 1}\left|Y_{t}\right|^{2}\right)^{1 / 2}$. Define the mapping $\Phi$ on $S^{2}$ by letting $\Phi(Y)$ be the first component $X$ of the solution $(X, K)$ of the Skorokhod problem associated with $H^{\tau_{k}-}+$ $\int_{0}^{*} F(Y)_{s-} d Z_{s}^{\tau_{k}-}$. We will show that $\Phi$ is a contraction mapping on $S^{2}$. To see this, we first observe that $\Phi(Y \equiv 0)=H^{\tau_{k}-}+K^{\tau_{k}-}$, since $F(0) \equiv 0$. Hence

$$
E \sup _{t \leq 1}\left|\Phi(0)_{t}\right|^{2} \leq 2 E \sup _{t \leq 1}\left|H_{t}^{\tau_{k}-}\right|^{2}+2 E \sup _{t \leq 1}\left|K_{t}^{\tau_{k}-}\right|^{2} \leq 2 k^{2}+2 E \sup _{t \leq 1}\left|K_{t}^{\tau_{k}-}\right|^{2} .
$$

Therefore, by Corollary 2.4, $\Phi(Y) \in S^{2}$. Furthermore, by Remark 3.1, for any $Y, \widehat{Y} \in S^{2}$ we have

$$
\begin{aligned}
& E \sup _{t \leq 1}\left|\Phi(Y)_{t}-\Phi(\widehat{Y})_{t}\right|^{2} \\
& \leq C_{1}\left(E \int_{0}^{\tau_{k}-}\left\|F(Y)_{s-}-F(\widehat{Y})_{s-}\right\|^{2} d\left([M]_{s}+\langle M\rangle_{s}\right)\right. \\
&\left.+E\left(|B+J|_{\tau_{k}-} \cdot \int_{0}^{\tau_{k}-}\left\|F(Y)_{s-}-F(\widehat{Y})_{s-}\right\|^{2} d|B+J|_{s}\right)\right) \\
& \leq C_{1}\left(E \sup _{t \leq 1}\left\|F(Y)_{t-}-F(\widehat{Y})_{t-}\right\|^{2}\left([M]_{\tau_{k}-}+\langle M\rangle_{\tau_{k}-}\right)\right. \\
&\left.+E \sup _{t \leq 1}\left\|F(Y)_{t-}-F(\widehat{Y})_{t-}\right\|^{2}|B+J|_{\tau_{k}-}^{2}\right) \\
& \leq 6 C_{1} a^{2} E \sup _{t \leq 1}\left\|F(Y)_{t-}-F(\widehat{Y})_{t-}\right\|^{2} \\
& \leq 6 C_{1} a^{2} L^{2} E \sup _{t \leq 1}\left|Y_{t}-\widehat{Y}\right|^{2}=\frac{1}{2} E \sup _{t \leq 1}\left|Y_{t}-\widehat{Y}_{t}\right|^{2} .
\end{aligned}
$$

From the above we see that $\Phi: S^{2} \rightarrow S^{2}$ is a contraction. Hence, by the Banach contraction principle, it has a fixed point $X^{k}$, which is a unique 
solution of $(1.1)$ on $\left[0, \tau_{k}\left[\right.\right.$. Since $P\left(\tau_{k}=\tau\right) \uparrow 1$, putting $X=X^{k}$ on $\left[0, \tau_{k}\right.$ [ we obtain a unique solution on $[0, \tau[$. Moreover, putting

$$
X_{\tau}=\Pi\left(X_{\tau-}+\Delta H_{\tau}+\left\langle F(X)_{\tau-}, \Delta Z_{\tau}\right\rangle\right)
$$

we obtain a solution on $[0, \tau]$, because $F\left(X^{\tau-}\right)_{\tau-}=F(X)_{\tau-}$ by the definition of $F$.

Now, we define a sequence of stopping times

$$
\tau^{0}=\tau, \quad \tau^{k+1}=\tau^{k}+\inf \left\{t>0 ; \max \left([\bar{M}]_{t},|\bar{B}|_{t}^{2},|\bar{J}|_{t}^{2}\right)>a^{2}\right\} \wedge\left(\tau_{k}^{\prime} \wedge 1\right),
$$

where $\bar{M} .=M_{\tau^{k}+}-M_{\tau^{k}}, \bar{B} .=B_{\tau^{k}+}-B_{\tau^{k}}, \bar{J} .=J_{\tau^{k}+}-J_{\tau^{k}}, \bar{H}$. $=$ $H_{\tau^{k}+\cdot}-H_{\tau^{k}}, \tau_{k}^{\prime}=\inf \left\{t>0,\left|\bar{H}_{t}\right| \geq \operatorname{dist}\left(a^{\prime}, \partial D\right) / 2\right\}$. By what has been proved there exists a unique solution of (1.1) on $\left[0, \tau^{0}\right]$. By the same method as above, having a unique solution on $\left[0, \tau^{k}\right]$, we can construct a solution of (1.1) on $\left[0, \tau^{k+1}\right]$. Since $\tau^{k} \uparrow \infty$, the theorem follows.

Corollary 3.3. If $f: \mathbb{R}^{d} \rightarrow \mathbb{R}^{d} \otimes \mathbb{R}^{d}$ is Lipschitz then for every $\left(\mathcal{F}_{t}\right)$ adapted process $H$ with $H_{0} \in \bar{D}$ and every $\left(\mathcal{F}_{t}\right)$ adapted semimartingale $Z$ with $Z_{0}=0$ there exists a unique strong solution of the SDE

$$
X_{t}=H_{t}+\int_{0}^{t}\left\langle f\left(X_{s-}\right), d Z_{s}\right\rangle+K_{t}, \quad t \in \mathbb{R}^{+} .
$$

Acknowledgements. I would like to thank Prof. Leszek Słomiński for suggesting the problem and for many stimulating conversations.

\section{References}

[1] S. V. Anulova and R. Sh. Liptser, Diffusional approximation for processes with the normal reflection, Theory Probab. Appl. 35 (1990), 411-423.

[2] E. Cépa, Problème de Skorohod multivoque, Ann. Probab. 26 (1998), 500-532.

[3] A. Jakubowski, J. Mémin and G. Pagés, Convergence en loi des suites d'intégrales stochastiques sur l'espace $\Delta^{1}$ de Skorokhod, Probab. Theory Related Fields 81 (1989), 111-137.

[4] J. L. Menaldi, Stochastic variational inequality for reflected diffusion, Indiana Univ. Math. J. 32 (1983), 733-744.

[5] P. E. Protter, On the existence, uniqueness, convergence and explosions of solutions of systems of stochastic integral equations, Ann. Probab. 5 (1977), 243-261.

[6] —, Stochastic Integration and Differential Equations, Springer, Berlin, 1990.

[7] A. V. Skorokhod, Stochastic equations for diffusion processes in a bounded region 1, 2, Theory Probab. Appl. 6 (1961), 264-274, 7 (1962), 3-23.

[8] L. Słomiński, On approximation of solutions of multidimensional SDE's with reflecting boundary conditions, Stochastic Process. Appl. 50 (1994), 197-219.

[9] - On the $L^{p}$-distance between semimartingales reflecting in different domains, Stoch. Stoch. Rep. 71 (2000), 91-118.

[10] A. Storm, Stochastic differential equations with a convex constraint, ibid. 53 (1995), $241-274$. 
[11] H. Tanaka, Stochastic differential equations with reflecting boundary condition in convex regions, Hiroshima Math. J. 9 (1979), 163-177.

Weronika Łaukajtys

Faculty of Mathematics and Computer Science

Nicolaus Copernicus University

Chopina $12 / 18$

87-100 Toruń, Poland

E-mail: vera@mat.uni.torun.pl

Received May 5, 2004;

received in final form October 6, 2004 